\title{
Secondary Erosive Arthritis in a Young Lady - A Rare Manifestation of Primary Antiphospholipid Antibody Syndrome
}

\author{
Sathish Muthu ${ }^{1,4,5}$, Girinivasan Chellamuthu ${ }^{1}$, Thiruvenkita Prasad Gopalsamy², \\ Velmurugan Kandasamy ${ }^{3}$
}

Learning Point of the Article:

Secondary erosive arthritis of knee as a rare presentation of primary anti-phospholipid antibody syndrome must be kept in mind and its treatment involves multidisciplinary approach for its effective management.

\section{Abstract}

Introduction: Arthritis in primary antiphospholipid antibody syndrome (PAPS) is a rare manifestation that is much more common in secondary antiphospholipid antibody syndrome (APS), particularly those associated with systemic lupus erythematosis (SLE), and has been reported to be non-erosive responding to conservative management. In this background, we describe a case of secondary erosive arthritis of knee (SEAK) in a female patient with PAPS.

Case Report: Thirty-seven-year-old working women presented with chronic right knee pain for the past 2 years which was increasing in severity and interfering with her activities of daily living for the past 3 months. The patient was a known case of PAPS with a history of one early and one late abortion. On radiological examination, Grade IV secondary osteoarthritis knee was made out. The patient underwent total knee replacement. At 2 years follow-up, the patient had a good functional outcome. To the best of our knowledge, this is the first report of secondary osteoarthritis in PAPS requiring arthroplasty. Perioperative management is crucial in PAPS to prevent thromboembolic complications. Multimodality approach with strict patient compliance is a key to achieve good functional recovery.

Conclusion: SEAK can be a rare presentation of PAPS. Secondary causes like SLE or rheumatoid arthritis must be ruled out before a diagnosis of PAPS is made. Perioperative management in APS is critical and challenging. Multidisciplinary team approach involving internal medicine, anesthesiology, orthopedics, and rehabilitative departments is essential.

Key words: Antiphospholipid syndrome, arthritis, knee replacement arthroplasty.

\section{Introduction}

Primary antiphospholipid antibody syndrome (PAPS) also known as Hughes syndrome is characterized by venous or arterial thromboembolic manifestations or miscarriages orchestrated by the presence of one or more antiphospholipid antibodies [1]. Although these are the typical manifestations of the syndrome, it is essentially a multisystem disease of varying severity with a spectrum from sudden multiorgan failure catastrophic antiphospholipid antibody syndrome (CAPS) to subtle manifestations such as thrombophlebitis, livedo reticularis, transient ischemic attack, hemolytic anemia, skin ulcers, and pseudovasculitic skin lesions $[1,2,3]$.
The most common musculoskeletal manifestation is arthralgia [4]. The other rare manifestations are arthritis, avascular necrosis of bones, bone marrow necrosis presenting as hemolytic emergency, muscle infarctions, non-traumatic stress fractures, and osteoporosis. Arthritis in PAPS is a rare manifestation that is much more common in secondary antiphospholipid antibody syndrome (APS), particularly those associated with systemic lupus erythematosis (SLE), and has been reported to be non-erosive responding to conservative management $[4,5,6]$. In this background, we describe a case of secondary erosive arthritis of knee (SEAK) in a female patient with PAPS.

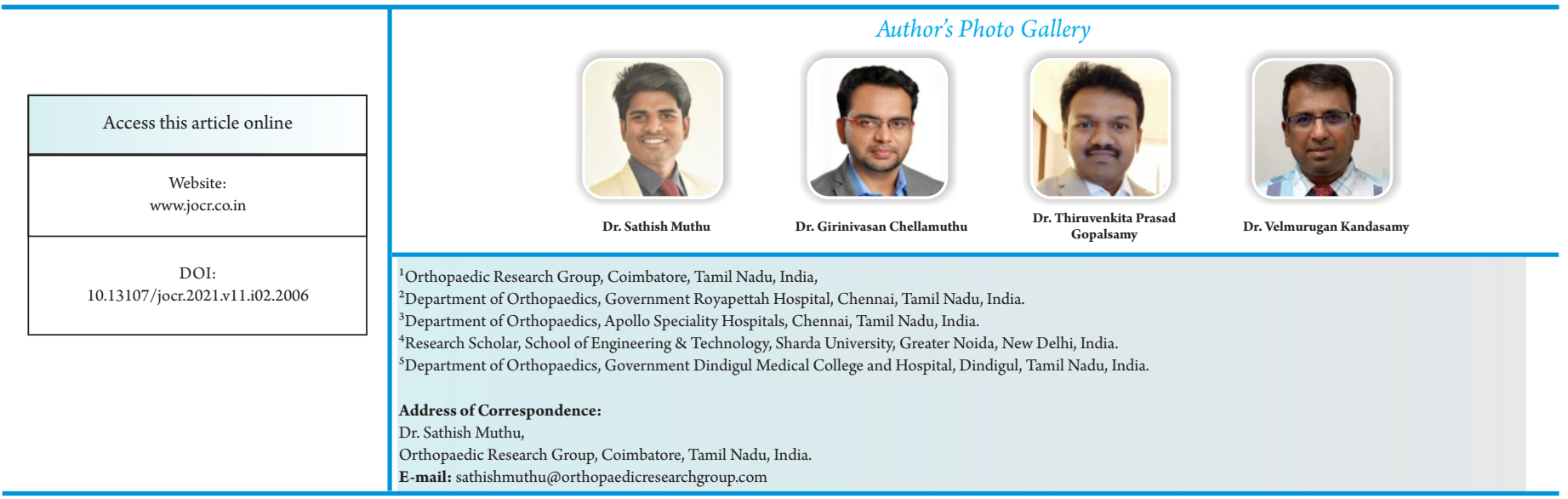

Journal of Orthopaedic Case Reports | pISSN 2250-0685 | eISSN 2321-3817 | Available on www.jocr.co.in | doi:10.13107/jocr.2021.v11.i02.2006 This is an Open Access article distributed under the terms of the Creative Commons Attribution Non-Commercial License (http://creativecommons.org/licenses/by-nc/3.0) which permits unrestricted non-commercial use, distribution, and reproduction in any medium, provided the original work is properly cited. 


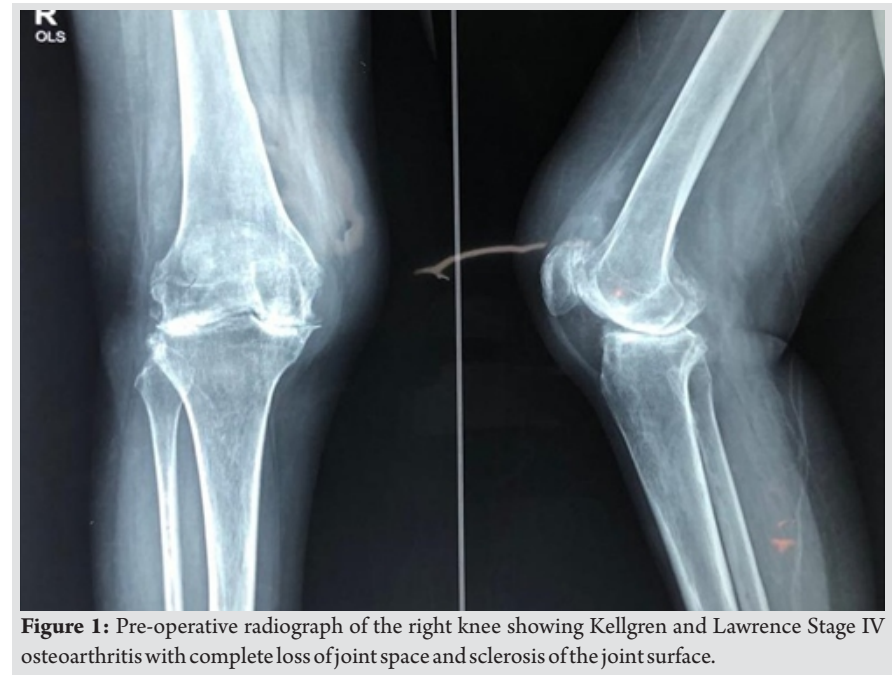

Case Report

Thirty-seven-year-old working women presented with chronic right knee pain for the past 2 years which was increasing in severity and interfering with her activities of daily living for the past 3 months. The patient was a known case of PAPS with obstetric history of one early pregnancy loss ( 8 weeks) and a second trimester pregnancy loss of an otherwise morphologically normal fetus.

On examination, the right knee was warm with moderate effusion. The range of movement was $10-90^{\circ}$ with a fixed flexion deformity of $10^{\circ}$. Movements were associated with pain and joint crepitus. Patellofemoral movements were painful. Distal pulses were felt equally on both sides.

Clinical markers for SLE, rheumatoid arthritis (RA), and Sjogren's syndrome indicating secondary APS were absent.

The patient had high anticardiolipin and anti- $\beta 2$-glycoprotein antibody confirming the diagnosis of APS (double positive). She was negative for antinuclear antibodies panel (anti-doublestranded DNA, anti-Smith, anti-U1RNP, anti-Ro/SSA, and anti-La/SSB), anti-CCP antibodies, and rheumatoid factor. Her blood counts, urine routine tests, and liver and renal function tests were within normal limits. ESR and CRP were slightly elevated.

Radiography of the right knee showed Kellgren and Lawrence Stage IV osteoarthritis [7] of the knee with complete loss of joint space and sclerosis of the joint surface and valgus knee, as shown in (Fig. 1). Hence, a diagnosis of SEAK with PAPS was made.

To provide a pain-free mobile joint, we decided to do total knee replacement (TKR). Hematologist and physician expert opinion were sought. The patient was already on low-dose aspirin (LDA) of $100 \mathrm{mg}$. We decided to continue the LDA. We added a prophylactic dose of low-molecular-weight heparin

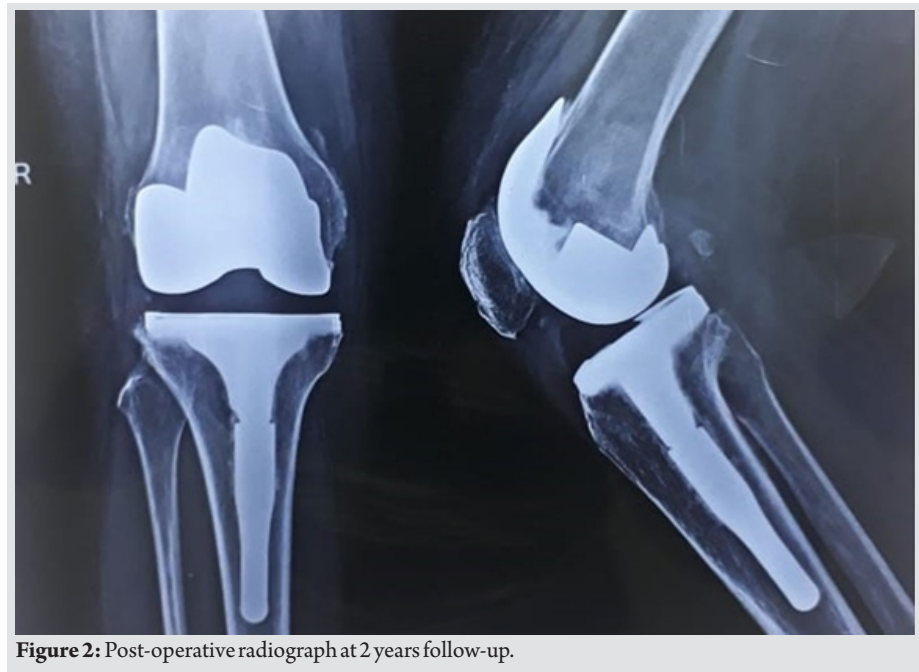

(LMWH) which we stopped $12 \mathrm{~h}$ before TKA. The patient underwent TKR following the standard operative protocols. LMWH was restarted $12 \mathrm{~h}$ after the procedure. The patient was on intermittent pneumatic compression (IPC) device perioperatively.

The patient was under regular follow-up for 2 years post-surgery with good radiological outcome, as shown in (Fig. 2). The range of motion was full and free form $0-100^{\circ}$ with an Oxford Knee Score of 44 indicating satisfactory joint function.

\section{Discussion}

APS is an autoimmune hypercoagulable state characterized by the presence of one or more of the antiphospholipid antibodies (APA) - anticardiolipin antibodies, anti-beta glycoprotein antibodies, and lupus anticoagulant [1]. Every organ can be a target of APS [3]. The diagnosis of APS is made when any one of the laboratory and one clinical revised Sapporo criteria was met. The clinical criteria include ( 1 ) three or more early ( $<10$ weeks) pregnancy loss or one or more late loss of a morphologically normal fetus and (2) venous or arterial thromboembolic event. The laboratory criteria include presence of one or more APA $[8$, $9,10]$. APS can be primary or secondary to conditions like SLE or RA.

The orthopedic manifestations of APS are not usually clinically significant to warrant an orthopedic consultation and are successfully managed with analgesics and immunosuppressants used to treat the primary pathology [4]. Rare events like avascular necrosis warrant orthopedic intervention [10]. Severe secondary arthritis of joints requiring joint replacement surgeries has not yet been reported in the literature. This is the first such report to our best knowledge which was essentially a diagnosis of exclusion as we ruled out other secondary etiologies.

Management of SEAK in APS has its own challenges [11]. Perioperative management is critical [12]. Surgery can trigger 
thrombosis (second hit hypothesis) or even CAPS $[13,14,15]$. The first step in management is to analyze the antibody profile of APS. The presence of lupus anticoagulant or high titers of any of the two APA shows a high-risk antibody profile with an increased risk of thromboembolic complications [11, 16, 17]. Being a rare condition, the literature on standard perioperative management guidelines in APS is lacking [11].

Saunders et al. [12] stratified procedures into high-, moderate-, and low-risk groups. Orthopedic interventions are classified under high-risk category. Surgery can precipitate CAPS. Hence, extreme caution should be excised in their management and a multidisciplinary team approach involving internal medicine, anesthesiology, orthopedics, and rehabilitative departments is essential.

Preoperatively, anticoagulation therapy in the form of $\mathrm{LMWH}$ till the day before surgery is essential. Counseling should be given to the patient regarding plan of care and need for longterm compliance.

Intraoperatively, epidural catheters should be avoided. The use of lower limb for venous access is restricted. Vascular interventions to monitor anesthetic parameters should be avoided [12]. The use of tourniquets is avoided. Measures to decrease blood loss like use of tranexamic acid are recommended. Physical devices to prevent venous pooling like IPC devices or gradual compression stockings should be used in the opposite limb [18]. Combining the benefits of both physical and pharmacological anticoagulation measures is essential in PAPS management [19].

Postoperatively, anticoagulants should be started at the earliest. Period without anticoagulation should be kept minimal. When warfarin is being considered, bridging with LMWH till its onset of action is essential. INR should be monitored. Active mobilization should be encouraged. Physical devices like IPC should be continued until the patient is actively mobilized [20]. APS is one disease where bleeding and thrombosis can coexist. Bleeding can be due to excessive anticoagulants, thrombocytopenia, or high-affinity antiprothrombin antibodies $[9,15,20]$. Hence, internal bleeding should be watched for and managed.

The presence of lupus anticoagulant alters the activated partial thromboplastin time (aPTT) test. Hence, factor Xa assay should be used to monitor the levels of anticoagulants instead of aPTT [12].

The other important aspect in perioperative management of PAPS is patient counseling. The patient must adhere to the anticoagulation prophylaxis to avoid any untoward complications due to the procedure and the disease.

\section{Conclusion}

SEAK can be a rare presentation of PAPS. Secondary causes like SLE or RA must be ruled out before a diagnosis of PAPS is made. Perioperative management in APS is critical and challenging. Multidisciplinary team approach involving internal medicine, anesthesiology, orthopedics, and

\section{Clinical Message}

Secondary erosive arthritis of knee as a rare presentation of PAPS must be kept in mind and its treatment involves multidisciplinary approach for its effective management.

\section{References}

1. Palomo I, Segovia F, Ortega C, Pierangeli S. Antiphospholipid syndrome: A comprehensive review of a complex and multisystemic disease. Clin Exp Rheumatol 2009;27:668-77.

2. Harris EN, Pierangeli SS. Primary, secondary, catastrophic antiphospholipid syndrome: Is there a difference? Thromb Res 2004;114:357-61.

3. Cervera R, Piette JC, Font J, Khamashta MA, Shoenfeld Y, Camps MT, et al. Antiphospholipid syndrome: Clinical and immunologic manifestations and patterns of disease expression in a cohort of 1,000 patients. Arthritis Rheum 2002;46:1019-27.

4. Noureldine MH, Khamashta MA, Merashli M, Sabbouh T, Hughes GR, Uthman I. Musculoskeletal manifestations of the antiphospholipid syndrome. Lupus 2016;25:451-62.

5. Vasoo S, Sangle S, Zain M, D’Cruz D, Hughes G. Orthopaedic manifestations of the antiphospholipid (Hughes) syndrome. Lupus 2005; 14:339-45.

6. Rueda JC, Duque MA, Mantilla RD, Iglesias-Gamarra A. Osteonecrosis and antiphospholipid syndrome. J Clin Rheumatol 2009; 15:130-2.

7. Kellgren JH, Lawrence JS. Radiological assessment of osteoarthrosis. Ann Rheum Dis 1957;16:494-502.

8. Wilson W, Gharavi A, Koike T, Lockshin MD, Branch DW, Piette JC, et al. International consensus statement on preliminary classification criteria for definite antiphospholipid syndrome: Report of an international workshop. Arthritis Rheum 1999;42:1309-11.

9. Miyakis S, Lockshin M, Atsumi T, Branch DW, Brey RL, Cervera $\mathrm{R}$, et al. International consensus statement on an update of the classification criteria for definite antiphospholipid syndrome (APS). J Thromb Haemost 
2006;4:295-306.

10. Rostom S, Bahiri R, Hajjaj-Hassouni N. Multifocal avascular necrosis revealing antiphospholipid syndrome. Clin Rheumatol 2008;27:271-2.

11. Tektonidou MG, Andreoli L, Limper M, Amoura Z, Cervera R, Costedoat-Chalumeau N, et al. EULAR recommendations for the management of antiphospholipid syndrome in adults. Ann Rheum Dis 2019;78:1296-304.

12. Saunders KH, Erkan D, Lockshin MD. Perioperative management of antiphospholipid antibody-positive patients. Curr Rheumatol Rep 2014; 16:426.

13. Erkan D, Lockshin M. New approaches for managing antiphospholipid syndrome. Nat Clin Pract Rheumatol 2009;5:160-70.

14. Fischetti F, Durigutto P, Pellis V, Debeus A, Macor P, Bulla R, et al. Thrombus formation induced by antibodies to beta 2glycoprotein $\mathrm{I}$ is complement dependent and requires a priming factor. Blood 2005; 106:2340-6.

15. Asherson RA, Cervera R, Piette JC, Shoenfeld Y, Espinosa G, Petri MA, et al. Catastrophic antiphospholipid syndrome: Clues to the pathogenesis from a series of 80 patients. Medicine (Baltimore) 2001;80:355-76.
Conflict of Interest: Nil

Source of Support: Nil

Consent: The authors confirm that informed consent was obtained from the patient for publication of this case report

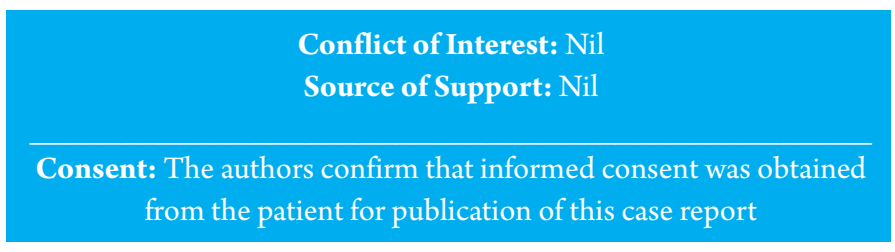

16. Ruiz-Irastorza G, Cuadrado MJ, Ruiz-Arruza I, Brey R, Crowther M, Derksen R, et al. Evidence-based recommendations for the prevention and long-term management of thrombosis in antiphospholipid antibodypositive patients. Lupus 2011 ;20:206-18.

17. Garcia D, Erkan D. Diagnosis and management of the antiphospholipid syndrome. N Engl J Med 2018;378:2010-21.

18. Chouhan VD, Comerota AJ, Sun L, Harada R, Gaughan JP, Rao AK. Inhibition of tissue factor pathway during intermittent pneumatic compression: A possible mechanism for antithrombotic effect. Arterioscler Thromb Vasc Biol 1999; 19:2812-7.

19. Kakkos SK, Caprini JA, Geroulakos G, Nicolaides AN, Stansby GP, Reddy DJ. Combined intermittent pneumatic leg compression and pharmacological prophylaxis for prevention of venous thromboembolism in high-risk patients. Cochrane Database Syst Rev 2008;4:CD005258.

20. Erkan D, Leibowitz E, Berman J, Lockshin MD. Perioperative medical management of antiphospholipid syndrome: Hospital for special surgery experience, review of literature, and recommendations. J Rheumatol 2002;29:843-9.

\section{How to Cite this Article}

Muthu S, Chellamuthu G, Gopalsamy TP, Kandasamy V. Secondary Erosive Arthritis in a Young Lady - A Rare Manifestation of Primary Antiphospholipid Antibody Syndrome. Journal of Orthopaedic Case Reports 2021 February; 11(2): 15-18. 